\title{
Macroscopic and microscopic characterisation of colitis in a mice model of inflammatory bowel disease and its application to nutritional therapeutics
}

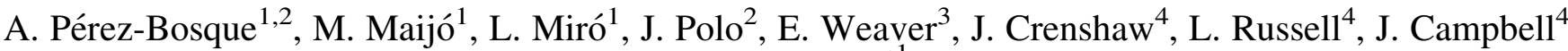 \\ and M. Moretó ${ }^{1}$ \\ ${ }^{1}$ Department of Physiology, Research Institute for Nutrition and Food Safety, Faculty of Pharmacy, University of Barcelona, \\ Spain, ${ }^{2}$ APC Europe, Granollers, Spain, ${ }^{3}$ Proliant Health and Biologicals, Ankeny, IA, USA and ${ }^{4}$ APC Inc., Ankeny,
}

IA, USA

\begin{abstract}
The mdr1a-I - mice lack the multiple drug resistance gene for P-glycoprotein, normally expressed in multiple tissues including intestinal epithelial cells ${ }^{(1)}$, and develop a spontaneous colitis. The degree of severity of the pathology and the time of appearance of colitis signs strongly depend on housing conditions. In the present study, we have carried out two experiments. The first experiment consisted of keeping mice in specific pathogen-free (SPF) conditions followed by conventional housing, in order to define conditions where food intake and growth were not markedly affected. In the second experiment, we examined the effects of spray-dried plasma (SDP) and an immunoglobulin concentrate (IC) supplementation on colitis markers in this mouse model of spontaneous colitis. Knock out (KO) mice (obtained from Taconic, $n=6-7$ ) were grown and maintained in SPF conditions until week 4 when they were transferred to conventional housing for 4 additional weeks. Wild-type (WT; $n=6-7)$ FVB $(+/+)$ mice were raised in conventional housing for the same period. The disease activity index (DAI) was defined accounting for changes in motor activity, body weight, consistency of faeces and presence of blood in stools. After killing, the weight of the colon, mesenteric lymph nodes and spleen were recorded. Interferon- $\gamma$ (IFN- $\gamma$ ) from mucosa of colon was measured by Bioplex cytokine assay. The permeability of colon mucosa was analysed ex vivo by confocal microscopy using FITC-Dextran $4 \mathrm{kDa}$ as marker. When dietary treatment was assayed, animals were supplemented with SDP (8\%, w/w), IC $(4 \%, w / w)$ or a control diet supplemented with milk proteins. Diets were given from day 19 (weaning) until day 56 . The disease onset were about 5-week-old when DAI progressively increased reaching a value of $1.94 \pm 0.23$ at day 58 (DAI in WT was $0.31 \pm 0.18$; $P<0.05$ ). In KO mice, the weight of colon, spleen and mesenteric lymph nodes was between 140 and $250 \%$ higher than in WT animals $(P<0.05)$. The permeability of crypts to dextran in KO was 2 -fold that in WT animals $(P<0.05)$. Mucosal production of IFN- $\gamma$ in the colon in the KO group $(1.7 \pm 0.2 \mathrm{pg} / \mathrm{mg}$ protein) was much higher than in the WT group $(0.3 \pm 0.04 \mathrm{pg} / \mathrm{mg} \mathrm{protein} ; P<0.05)$. Plasma supplements did not modify the DAI. However, they prevented in part the increase in colonic epithelial permeability and colonic hypertrophy in the mdr1a $-/-$ mice $(P<0.05)$. The protocol used in this study resulted in the development of mild colitis without affecting food intake and growth, though several markers such as IFN- $\gamma$ expression and crypt permeability were enhanced in the mdr1a $-/-$ animals. Preliminary results with supplemented diets indicate that this protocol is adequate to carry out studies on the effect of diets in the prevention of inflammatory bowel disease.
\end{abstract}

This work was supported by grant RDITCRD07-1-008 (Generalitat de Catalunya, Spain).

1. Wilk JN, Bilsborough J \& Viney JL (2005) Immunol Res 31, 151-159. 